\title{
Influence of pairing correlations on the radius of neutron-rich nuclei
}

\author{
Ying Zhang* \\ Department of Physics, School of Science, Tianjin University, Tianjin 300072, China \\ Ying Chen \\ Institute of Materials, China Academy of Engineering Physics, Mianyang 621700, China
}

Jie Meng

State Key Laboratory of Nuclear Physics and Technology, School of Physics, Peking University, Beijing 100871, China; School of Physics and Nuclear Energy Engineering, Beihang University, Beijing 100191, China; and Department of Physics, University of Stellenbosch, Stellenbosch, South Africa

Peter Ring

State Key Laboratory of Nuclear Physics and Technology, School of Physics, Peking University, Beijing 100871, China and Fakultät für Physik, Technische Universität München, D-85748 Garching, Germany

(Received 18 July 2016; revised manuscript received 23 September 2016; published 13 January 2017)

\begin{abstract}
The influence of pairing correlations on the neutron root mean square (rms) radius of nuclei is investigated in the framework of self-consistent Skyrme Hartree-Fock-Bogoliubov calculations. The continuum is treated appropriately by the Green's function techniques. As an example the nucleus ${ }^{124} \mathrm{Zr}$ is treated for a varying strength of pairing correlations. We find that, as the pairing strength increases, the neutron rms radius first shrinks, reaches a minimum, and beyond this point it expands again. The shrinkage is due to the the so-called pairing antihalo effect, i.e., due to the decrease of the asymptotic density distribution with increasing pairing. However, in some cases, increasing pairing correlations can also lead to an expansion of the nucleus due to a growing occupation of so-called halo orbits, i.e., weakly bound states and resonances in the continuum with low- $\ell$ values. In this case, the neutron radii are extended just by the influence of pairing correlations, since these halo orbits cannot be occupied without pairing. The term "antihalo effect" is not justified in such cases. For a full understanding of this complicated interplay, self-consistent calculations are necessary.
\end{abstract}

DOI: 10.1103/PhysRevC.95.014316

\section{INTRODUCTION}

Superfluidity is a quantum phenomenon found in various systems such as liquid helium, superconductors, atomic nuclei, and neutron stars. Nuclear superfluidity is caused by pairing correlations, induced by the attractive effective interaction between pairs of nucleons. This leads to an odd-even staggering in nuclear masses and separation energies, and to a considerable reduction of the moments of inertia in rotational bands. These phenomena are observed throughout the entire periodic table [1]. In the past two decades, exotic nuclei with large proton or neutron excess have been extensively discussed and new phenomena have been discovered such as proton radioactivity close to the proton drip line or neutron halos in some nuclei at the neutron drip line. The coupling to the continuum plays an essential role in these weekly bound systems [2-6]. A famous case is the nucleus ${ }^{11} \mathrm{Li}$, where the first neutron halo has been observed [7]. Without pairing correlations the two neutrons in the halo would not be bound to the ${ }^{9} \mathrm{Li}$ core. In these nuclei close to the neutron drip line the Fermi energy approaches the continuum threshold, and pairing correlations make it possible for neutrons to occupy not only the weakly bound orbits but also unbound orbits

*yzhangjenp@tju.edu.cn with very low orbital angular momentum $\ell=0$ or $\ell=1$ near the Fermi energy in the single-particle spectrum [8]. This could be most easily seen in the canonical basis, where the Hartree-Fock-Bogoliubov (HFB) wave function can be represented in the form of a BCS state, as discussed in detail in Refs. [4-6,9]. Their wave functions can extend far outside the nucleus due to the low centrifugal barrier, which is crucial to the formation of the halo structure [4].

Of course, there is also the possibility to form a halo without pairing. If, for instance, the last occupied neutron orbit has an orbital angular momentum $\ell=0$ and a single-particle energy $\varepsilon$ just below the continuum threshold, in the asymptotic region the dominant contribution to the Hartree-Fock (HF) density of this nucleus has the form

$$
\rho_{\mathrm{HF}}(r) \propto \frac{\exp (-2 \kappa r)}{r^{2}} \text { for } r \rightarrow \infty
$$

with $\kappa=\sqrt{2 m|\varepsilon|} / \hbar$. The mean square neutron radius calculated with this density behaves as

$$
\left\langle r^{2}\right\rangle_{\mathrm{HF}} \propto \frac{1}{|\varepsilon|} .
$$

It diverges for $\varepsilon \rightarrow 0$.

Bennaceur et al. [10] showed that pairing correlations, leading to a finite pairing gap $\Delta$, prevent such a divergence. The mean square radius calculated with the asymptotic HFB 
density behaves as

$$
\left\langle r^{2}\right\rangle_{\mathrm{HFB}} \propto \frac{1}{\Delta} .
$$

Therefore, they concluded that the additional pairing binding energy acts against a development of an infinite root mean square (rms) radius that characterizes $\ell=0$ mean-field eigenfunctions in the limit of vanishing binding energy. This is then called the "pairing antihalo effect."

If one defines a "halo" by a divergence in the rms radius, this conclusion is definitely mathematically correct. However, in nature we have to consider additional points: first, experimentally observed halos [7] have a large but finite rms radius; second, apart from closed shells the coupling to the continuum causes and enhances pairing correlations. Then the proper mean-field description of the nucleus is given by the HFB theory. In order to understand the structure of the HFB wave function, it would be useful to represent it as a BCS state in its canonical basis [11], i.e., in terms of eigenstates of the density matrix, whose energies are defined as expectation values of the single-particle Hamiltonian $\hat{h}$ in the HFB equation. Then one could find clearly that there are not only contributions to the radius from the canonical orbits below the continuum threshold of $\hat{h}$, but also from partially occupied states with energies in the continuum. For orbits without or with small centrifugal barriers, i.e., for $s$ or $p$ levels their contributions are not negligible [4]. The coupling to those states definitely grows with increasing pairing correlations. If a halo could be formed only in cases without pairing, i.e., without coupling to the continuum, the three limiting conditions_zero pairing, $\varepsilon \rightarrow 0$, and low- $\ell$ values-would be actually difficult to meet in real nuclei due to the shell structure. Neutron halo phenomena would be very rare and rather accidental among the known neutron drip-line nuclei.

The influence of pairing correlations on single-particle configurations in the continuum has been studied in the literature [12]. However, these investigations were based on a fixed potential of Woods-Saxon shape and the spatial extension of the density was modified by changing artificially the depth of this potential. The continuum was taken into account by solving the HFB equation in a finite box of radius $R$.

It is the goal of this investigation to go a step further with respect to Ref. [12] and to clarify in a fully self-consistent way the influence of pairing correlations on the extension of neutron radii in these nuclei close to the drip line, as observed in experiments (for instance in Ref. [7]). It is certainly an interesting question to distinguish between the formation of a neutron skin or a neutron halo, as has been done in several theoretical investigations [13-15]. However, it is not the goal of the present work to go into such detail, as long as there are no precise experimental data available on the density distributions to distinguish these two phenomena. In light of the above considerations it is evident that the complicated interplay of the different phenomena of changing mean fields and pairing fields can only be achieved in fully self-consistent calculations with a proper treatment of the continuum. We will consider not only the influence of pairing on the asymptotic behavior of the wave functions of occupied, weakly bound, low $-\ell$ orbits, but also the role of the occupation probabilities introduced by the scattering of pairs around the Fermi energy and the coupling to the continuum in these loosely bound superfluid systems. Recently, covariant density functional theory has been used to study such phenomena with a discretized continuum [9]. Since, worldwide, nonrelativistic density functional theory is one of the most successful approaches in the description of exotic nuclei [16] we concentrate here on investigations based on density dependent HFB theory with Skyrme forces. A recently developed code using the Green's function method [17,18] allows us to avoid the discretization of the continuum and to solve the continuum HFB equations with the proper asymptotic behavior. Technical details of this method can be found in Ref. [19].

\section{TECHNICAL DETAILS}

As an example we consider the nucleus ${ }^{124} \mathrm{Zr}$, which has been predicted to be a neutron halo nucleus by relativistic continuum Hartree-Bogoliubov (RCHB) calculations [5,20]. Similar results have been reproduced by the Skyrme HFB theory [21,22]. We use the Skyrme functional SkI4, which has been carefully adjusted to the isospin properties of nuclear skins [23]. It is therefore used in many applications for the description of halo phenomena in the framework of nonrelativistic density functional theories, as in Refs. [21,22,24]. For the pairing force we use the density-dependent delta interaction (DDDI) discussed in Refs. [25,26]:

$$
v_{q}^{\text {pair }}\left(\boldsymbol{r}, \boldsymbol{r}^{\prime}\right)=\frac{1}{2}\left(1-P_{\sigma}\right) V_{q}(\boldsymbol{r}) \delta\left(\boldsymbol{r}-\boldsymbol{r}^{\prime}\right) \quad(q=n, p),
$$

where $V_{q}(\boldsymbol{r})$ is the pairing interaction strength. It is a function of the neutron and proton densities as

$$
V_{q}(\boldsymbol{r})=\eta V_{0}\left[1-x\left(\frac{\rho_{q}(\boldsymbol{r})}{\rho_{c}}\right)^{\alpha}\right]
$$

In order to avoid the well-known ultraviolet divergencies, we work in a pairing window, i.e., the quasiparticle space considered in these calculations is truncated by the maximal orbital angular momentum $l=12 \hbar$ and to the maximal quasiparticle energy $E_{\text {cut }}=60 \mathrm{MeV}$. The parameters in Eq. (5) are adopted as $V_{0}=-458.4 \mathrm{MeV} \mathrm{fm}^{-3}, x=0.71, \alpha=0.59$, and $\rho_{c}=0.08 \mathrm{fm}^{-3}$. The parameter $V_{0}$ representing the strength of the pairing force in free space is chosen to reproduce the scattering length $a=-18.5 \mathrm{fm}$ of the bare nucleon-nucleon interaction in the ${ }^{1} S_{0}$ channel [26]. In order to study the influence on pairing properties, we use in the following investigations the additional factor $\eta$ which can change the pairing strength.

The contour integration path $C$ used for the calculation of the total densities by the Green's function method of Ref. [19] is chosen to be a rectangle with the height $\gamma=0.1$ $\mathrm{MeV}$ and the length $E_{\text {cut }}=60 \mathrm{MeV}$. The energy step of the contour integration is $\Delta E=0.01 \mathrm{MeV}$. For comparison, we also perform HFB calculations by the box-discretized approximation, in which the HFB equation is solved with box boundary conditions [4,27]. Both the box-discretized and the continuum HFB calculations are performed with a box size $R_{\text {box }}=20 \mathrm{fm}$, and a mesh size $\Delta r=0.1 \mathrm{fm}$. 


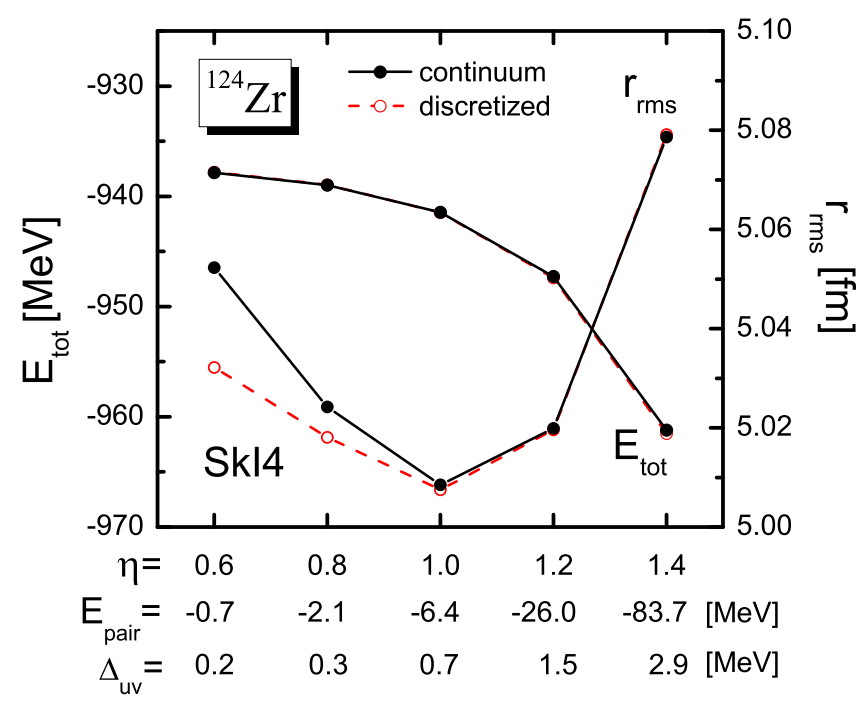

FIG. 1. Total energy $E_{\text {tot }}$ and neutron root mean square (rms) radius $r_{\text {rms }}$ obtained by the continuum (filled circle) and the discretized (open circle) Hartree-Fock-Bogoliubov (HFB) calculations for ${ }^{124} \mathrm{Zr}$ with the SkI4 interaction as a function of the strength $\eta V_{0}$ of the DDDI pairing force, where $V_{0}=-458.4 \mathrm{MeV} \mathrm{fm}^{-3}$.

\section{DISCUSSIONS}

Figure 1 shows the total energy $E_{\text {tot }}$ and the neutron rms radius $r_{\text {rms }}$ obtained by continuum and discretized HFB calculations for ${ }^{124} \mathrm{Zr}$ with varying DDDI pairing strengths $\eta V_{0}$, where $V_{0}=-458.4 \mathrm{MeV} \mathrm{fm}^{-3}$ and the factor $\eta=$ 0.6-1.4. For convenience, the corresponding neutron pairing energy $(-0.7$ to $-83.7 \mathrm{MeV})$ and the average pairing gap defined as

$$
\Delta_{u v}=\frac{\int \Delta(\boldsymbol{r}) \tilde{\rho}(\boldsymbol{r}) d \boldsymbol{r}}{\int \tilde{\rho}(\boldsymbol{r}) d \boldsymbol{r}}
$$

(0.2-2.9 MeV) are shown under the corresponding pairing strength factor $\eta$.

One can see that, as the pairing strength increases, the total energy $E_{\text {tot }}$ monotonically decreases; the nucleus becomes more bound, due to the attractive pairing interaction. Moreover, one can find almost no difference between the total energy obtained by the discretized and by the full continuum HFB calculations. Naively thinking, the corresponding nuclear size is shrinking as the nucleus becomes more bound. However, both the discretized and the continuum calculations show that the neutron rms radius first decreases, then reaches a local minimum at $\eta \approx 1.0$, and afterwards increases. The difference between the discretized and continuum results for the neutron rms radius is more obvious for weak pairing case with a shallow Fermi energy.

In order to understand the change of the rms radius as a function of the pairing strength, we first plot in Fig. 2 the total neutron density obtained by the continuum HFB calculation for the pairing strength factors $\eta=0.6,1.0$, and 1.4. A linear scale is used in the main figure for the inner and the surface region, and a logarithmic scale is used in the inset for the asymptotic region.

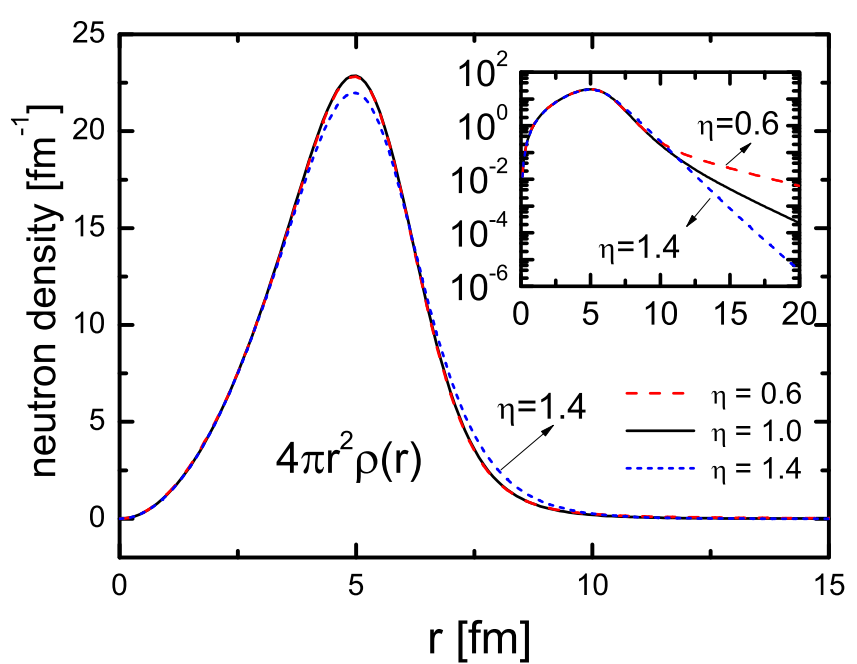

FIG. 2. Total neutron densities $4 \pi r^{2} \rho(r)$ of ${ }^{124} \mathrm{Zr}$ obtained by the continuum HFB calculation with the SkI4 interaction and the DDDI pairing force strengths $\eta V_{0}$ for $\eta=0.6$ (dashed line), 1.0 (solid line), 1.4 (dotted line). The inset shows the asymptotic behavior of the density in a logarithmic scale.

In the inset we see that as the pairing strength increases, the density in the asymptotic region $(r>10 \mathrm{fm})$ always decays faster. This corresponds to the antihalo effect discussed in Ref. [10] leading to a shrinkage of the rms radius. However, we also have to consider the change of the density inside the nucleus and at the surface. From $\eta=0.6$ to $\eta=1.0$, the density inside the nucleus and at the surface does not change much as shown in the main figure, but decays faster in the asymptotic region as shown in the inset. As a result, the total rms radius is decreasing up to $\eta=1.0$. When $\eta$ increases further, we observe a change of the density in the surface region: first a small decrease around $r \sim 5 \mathrm{fm}$ and then a small increase in the region between $6 \lesssim r \lesssim 10 \mathrm{fm}$. For radii $r \gtrsim 10 \mathrm{fm}$ the density decreases again as shown in the inset. However, the densities are so small at these large radii that this effect can only be recognized on the logarithmic scale and it does not contribute much to the total radius. Therefore, the total radius is determined by a competition between the increase of the density at the surface and the decrease in the asymptotic region. Although the density decays even faster in the asymptotic region, the increase at the surface $6 \lesssim r \lesssim$ $10 \mathrm{fm}$ finally produces an increase of the total rms radius for $\eta>1.0$.

In order to understand the reason why there is an increase of the density at the surface, we plot in Fig. 3 the information on important single-neutron levels in the vicinity of the Fermi energy as function of the pairing strength factor $\eta$. In the discretized method the total neutron rms radius of the nucleus is given as

$$
\left(r_{\mathrm{rms}}\right)^{2}=\frac{\sum_{n l j}(2 j+1) v_{n l j}^{2}\left(r_{n l j}^{\mathrm{rms}}\right)^{2}}{\sum_{n l j}(2 j+1) v_{n l j}^{2}} .
$$




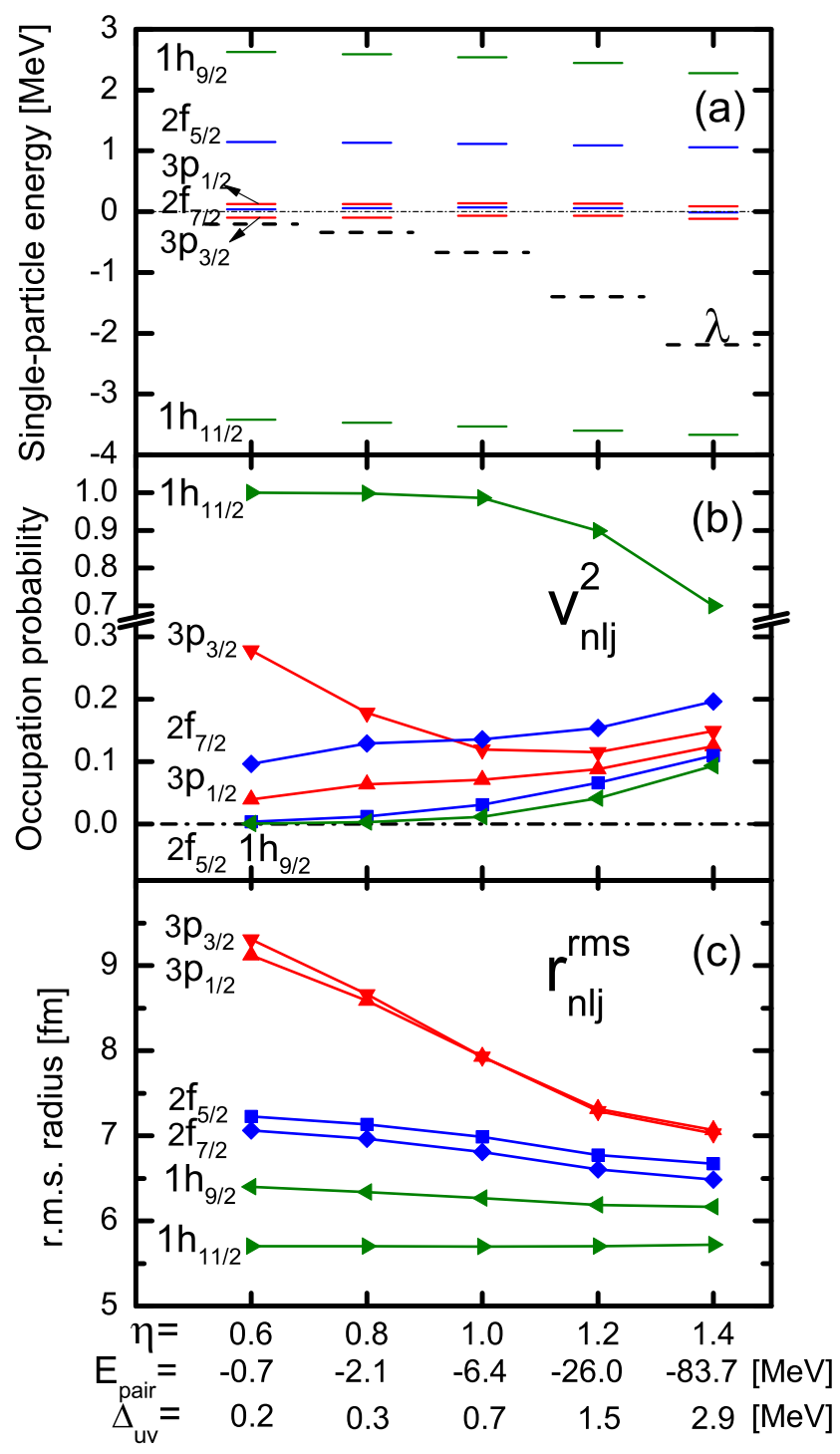

FIG. 3. (a) Single-neutron energies of ${ }^{124} \mathrm{Zr}$ around the Fermi energy for $3 p, 2 f$, and $1 h$ orbits, which are the eigenvalues of the single-particle Hamiltonian $h$ after the final convergence of the continuum Skyrme HFB calculation, and the dashed line denotes the Fermi energy $\lambda$; (b) neutron occupation probabilities $v_{n l j}^{2}$, and (c) the contributions to the rms radius $r_{n l j}^{\mathrm{rms}}$ from the quasiparticle states corresponding to the orbits shown in panel (a) within the energy $E=0-6 \mathrm{MeV}$ in the continuum HFB calculation obtained with different pairing strength $\eta V_{0}$.

It is determined not only by the rms radii $r_{n l j}^{\mathrm{rms}}$ of the individual orbits,

$$
r_{n l j}^{\mathrm{rms}}=\left(\frac{\int 4 \pi r^{4} \rho_{n l j}(r) d r}{\int 4 \pi r^{2} \rho_{n l j}(r) d r}\right)^{1 / 2}
$$

but also by the occupation factors $(2 j+1) v_{n l j}^{2}$ of these orbits,

$$
v_{n l j}^{2}=\frac{1}{2 j+1} \int 4 \pi r^{2} \rho_{n l j}(r) d r .
$$

Here the density distribution $\rho_{n l j}(r)$ of the orbit with the quantum numbers $(n l j)$ is given by the square of the corresponding quasiparticle wave function.

In our application the continuum is not discretized. We use the Green's functions techniques. Here the sum over $n$ in Eq. (7) is replaced by a contour integration in the complex energy plane containing all the bound states and the resonances. The contributions of the individual orbitals to the density is calculated by the HFB Green's function constructed by the quasiparticle wave functions as

$$
\rho_{n l j}(r)=\frac{1}{4 \pi} \frac{1}{2 \pi i}(2 j+1) \oint_{C_{n}} \frac{\mathcal{G}_{0, l j}^{(11)}(r, r, E)}{r^{2}} d E .
$$

The integration is carried out on a closed contour path $C_{n}$ [19], choosing for each pair of quantum numbers $(l j)$ a rectangular path with the energy interval $E=0-6 \mathrm{MeV}$. This path includes for each $(l j)$ value the contribution of the lowest quasiparticle state, i.e., the state closest to the Fermi energy, which is shown in Fig. 3.

In Fig. 3 we give details on some important single-neutron levels around the Fermi energy in ${ }^{124} \mathrm{Zr}$ as a function of the pairing strength factor $\eta$. In principle, to investigate the singleparticle properties, one should work in the canonical basis, where any HFB wave function can be expressed as a BCS wave function [11] and the occupation numbers $v_{n l j}^{2}$ are of BCS form. In this case $\varepsilon_{n l j}=\langle n l j|\hat{h}| n l j\rangle$ is just the expectation value of the single-particle operator $\hat{h}$ in this basis. In the usual investigations working with a fixed box radius $R$ [4,9] one has only discrete levels $\varepsilon_{n l j}$ and it is easy to find the canonical basis just by diagonalizing the density matrix $\rho_{n n^{\prime}}^{l j}$. In our method, since we construct only the local density by the contour integral over the quasiparticle energy as shown in Eq. (10), we do not have access to the canonical basis for the moment. Instead, we show in Fig. 3(a) the singleparticle energies obtained by diagonalizing the single-particle Hamiltonian $\hat{h}$ on a mesh with a finite box size. We use them as a reference to show the important levels around the Fermi energy in the present investigation [4,27]. In the canonical basis, the occupation numbers $v_{n l j}^{2}$ depend in a sensitive way on the pairing correlations and the position of the corresponding energy levels $\varepsilon_{n l j}$. But for the same reason, we instead use the neutron density $\rho_{n l j}(r)$, derived from the Green's function around the $n$th single-neutron state in Eq. (10), to calculate the occupation numbers in Eq. (9) shown in Fig. 3(b). They give a reference for the occupation situation of those important levels with different pairing strengths. Figure 3(c) shows the rms radius $r_{n l j}^{\mathrm{rms}}$ calculated by the same neutron density in Eq. (10).

In the single-neutron spectrum in panel (a), we find the weakly bound $3 p_{3 / 2}$ orbit near the Fermi energy, the $2 f_{7 / 2}$ and $3 p_{1 / 2}$ orbits just above the continuum threshold, and the $2 f_{5 / 2}$ and $1 h_{9 / 2}$ orbits higher above. Without pairing, we know that the last two neutrons in ${ }^{124} \mathrm{Zr}$ occupy the $3 p_{3 / 2}$ orbit. With increasing pairing this level is more and more depleted as shown in panel (b). Only for $\eta>1.2$ its occupation probability slightly increases. At $\eta=0.6$ the Fermi energy is located near this level with a rather small pairing energy $(-0.7 \mathrm{MeV})$. As the pairing strength increases, we find that 
the single-neutron energies remain almost unchanged except for a little decrease for the $1 h$ orbits. At the same time, the pair scattering becomes stronger, and brings more neutrons from the region below the Fermi energy to the region above it. As a result, the occupation of the $1 h_{11 / 2}$ level decreases and the Fermi energy is pulled down closer to this orbit. Panel (b) shows the occupation probabilities $v_{n l j}^{2}$ of the $3 p$, $2 f$, and $1 h$ orbits around the Fermi energy. Since the $s$ and $d$ orbits with positive parity in the continuum have a much smaller occupation probability $(<2 \%)$, they are not shown in this figure. One can see that, as the pairing strength increases, the neutrons in the $1 h_{11 / 2}$ orbits are scattered up to the $3 p_{1 / 2}$, $2 f_{7 / 2}$ orbits, and even to the $2 f_{5 / 2}$ and $1 h_{9 / 2}$ orbits high above in the continuum, while the neutron number for the $3 p_{3 / 2}$ orbit first decreases and then increases. Here, we should notice that the occupation probability calculated by Eq. (9) counts not only the neutrons located on the single-neutron levels, but also in the nearby continuum within the quasiparticle energy $E=0-6 \mathrm{MeV}$, since these states become quasiparticle resonant states with finite width due to the coupling with the continuum by pairing [19].

The rms radii in Eq. (8) for the corresponding orbits are shown in panel (c). One can clearly see that, due to the lower centrifugal barrier, the rms radii of the $3 p$ orbits are larger than those of the $2 f$ and $1 h$ orbits, especially when the pairing strength is small. As the pairing strength increases, due to the pairing antihalo effect, the rms radius decreases for the $3 p$ orbits dramatically, but it remains almost the same for the $1 h_{11 / 2}$ orbit. Thus at $\eta=1.4$ the rms radii of these levels become comparable with each other. The smaller rms radius with a stronger pairing gap seems to coincide with the so-called pairing antihalo effect shown by Eq. (3). However, we should keep in mind that the total neutron rms radius (7) is determined not only by the rms radii $r_{n l j}^{\mathrm{rms}}$ of the individual orbits in Eq. (8), but also by the occupation factors $(2 j+1) v_{n l j}^{2}$ of these orbits in Eq. (9). Therefore it can happen, as in the case of ${ }^{124} \mathrm{Zr}$, that all the individual $\mathrm{rms}$ radii, given in panel (c) of Fig. 3, decrease for $\eta>1.0$ and nonetheless the total rms radius shown in Fig. 1 is increasing. The total neutron density has contributions from all the levels weighted by the occupation probabilities shown in panel (b). As the pairing strength increases, the occupation probability of the $3 p_{1 / 2}$, $2 f_{7 / 2}$, and even $2 f_{5 / 2}$ as well as $1 h_{9 / 2}$ orbits higher up in the continuum increases around 0.1 respectively. This contributes to a larger total rms radius $r_{\text {rms }}$. As a result of the competition between the shrinkage of the rms radius $r_{n l j}^{\text {rms }}$ for the individual occupied single-particle orbits and the growing occupation $v_{n l j}^{2}$ of low- $\ell$ orbits with large rms radius in the continuum, the total rms radius first decreases and then increases as shown in Fig. 1. Strong pairing correlations do not necessarily shrink the nuclear size as indicated by the word "antihalo." It is essential to take into account the change of the occupation among different orbits due to the pair scattering, and especially the contributions from the continuum.

More explicitly, in Figs. 4(a)-4(f) we plot the neutron densities $4 \pi r^{2} \rho_{n l j}(r)$ calculated by Eq. (10) (including the degeneracy factor $2 j+1$ ) for $3 p, 2 f$, and $1 h$ orbits as a function of the pairing strengths factor $\eta$. Again, the inner

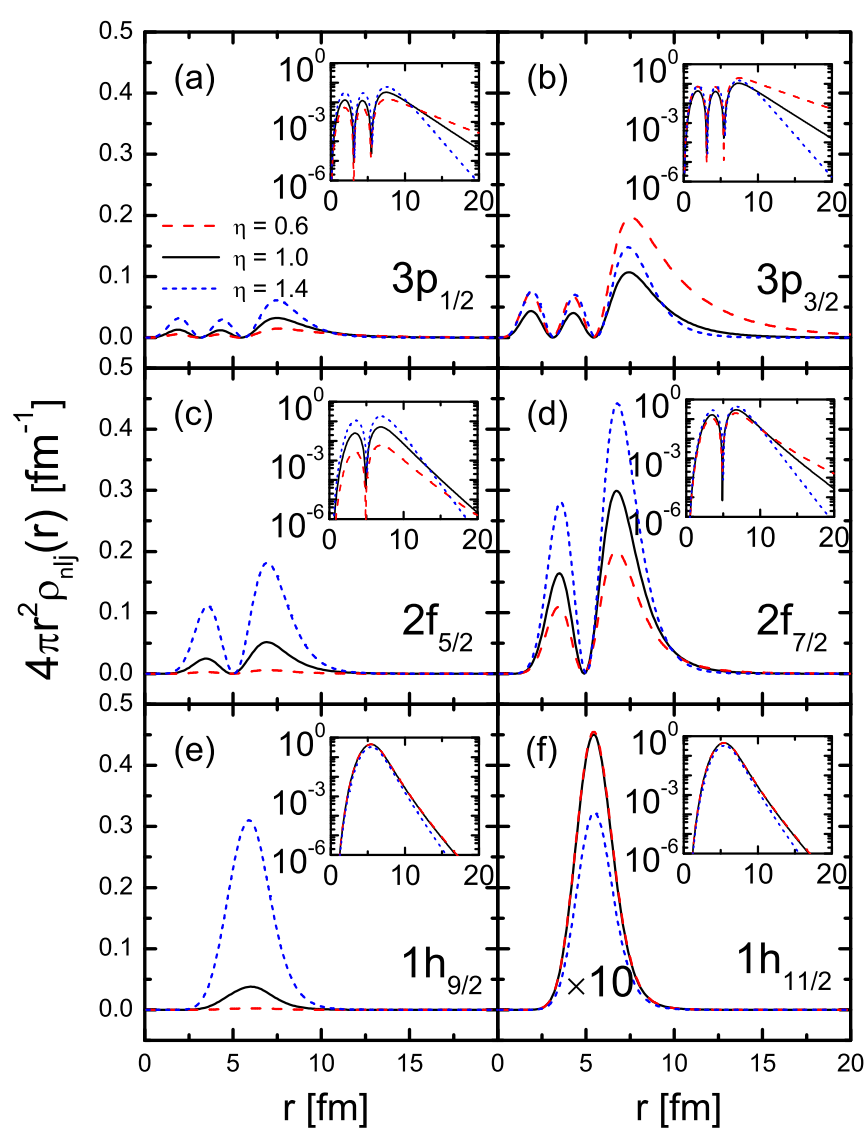

FIG. 4. Neutron density $4 \pi r^{2} \rho_{n l j}(r)$ of $3 p, 2 f$, and $1 h$ orbits in ${ }^{124} \mathrm{Zr}$ contributed from the quasiparticle states within the energy $E=$ 0-6 MeV calculated by the continuum HFB approach with different pairing strengths $\eta V_{0}$. The insets give the same density distributions with logarithmic scale. In panel (f) for the $1 h_{11 / 2}$ orbit, the neutron density is scaled by a factor 0.1 compared to the original value.

and surface parts of the densities are plotted in a linear scale in the main figures, and the asymptotic behavior is plotted in a logarithmic scale in the insets. For comparison, we plot the densities for different orbits in the same linear and logarithmic scales respectively, only the $1 h_{11 / 2}$ orbit in panel (f) is scaled by a factor 0.1 .

Obviously, the $1 h_{11 / 2}$ orbit contributes most to the density $\rho_{n l j}(r)$ within the quasiparticle energy interval $E=0-6 \mathrm{MeV}$ shown in panel (f). It remains almost the same from $\eta=0.6$ to $\eta=1.0$, but it drops dramatically for $\eta=1.4$ at the peak near $r \approx 5 \mathrm{fm}$, which leads to the reduction of the occupation probability shown in Fig. 3(b). Actually, this drop of the density at the peak is also the main reason for the decrease of the total density around $r \approx 5$ fm shown in Fig. 2 .

The contribution to the density $\rho_{n l j}(r)$ from the $3 p_{3 / 2}$ orbit in panel (b) decreases obviously at the surface between $\eta=0.6$ and $\eta=1.0$, which leads to the reduction of its occupation probability shown in Fig. 3(b). With further stronger pairing at $\eta=1.4$, the density at the surface increases again due to the neutrons scattered from below (e.g., from the $1 h_{11 / 2}$ orbit). In the asymptotic region, the density contribution always decays faster with increasing pairing strength for all values of the 
parameter $0.6 \leqslant \eta \leqslant 1.4$. Moreover, one should notice that it is the $3 p_{3 / 2}$ state that dominates the density in the asymptotic region and thus governs the asymptotic behavior of the total density for $r \gtrsim 10 \mathrm{fm}$ shown in the inset of Fig. 2.

Another large density contribution comes from the $2 f_{7 / 2}$ orbit in panel (d). It has a dramatic increase inside and around the surface, with stronger pairing strength. This helps to cancel the effect of the decreasing contribution from the $3 p_{3 / 2}$ orbit.

For the $3 p_{1 / 2}, 2 f_{5 / 2}$, and $1 h_{9 / 2}$ orbits shown in panels (a), (c), and (e), one finds an obvious increase from almost zero for the density contribution especially around the surface. This increase indicates that neutrons begin to occupy these orbits when the pairing scattering is strong enough. Together with the contribution from the $2 f_{7 / 2}$ orbit, we can explain the increase of the total density at the surface $(r=6-8 \mathrm{fm})$ for $\eta=1.4$ shown in Fig. 2, which at last leads to the increase of the total rms radius at $\eta=1.4$ shown in Fig. 1 .

So far, taking the nucleus ${ }^{124} \mathrm{Zr}$ as an example, we change the pairing strength by an arbitrary factor $\eta=0.6-1.4$, and see what happens to the neutron rms radius. Of course, the arbitrary change of the pairing strength is not physical, but it can serve as a model analysis of this problem. In order not to deviate from the physical point too much, we keep ourself not so far away from $\eta=1.0$. Actually, in Ref. [10], where the pairing antihalo effect is discussed, the rms radius derived from HF wave functions without pairing is compared with that derived from HFB wave functions with pairing. This is not exactly what we have done above. In order to compare with the no-pairing case, we also performed a HF calculation for this nucleus without pairing $(\eta=0)$, and obtain the neutron rms radius $r_{\mathrm{rms}}^{\mathrm{HF}}=5.08 \mathrm{fm}$, which is even $0.6 \%$ larger than the continuum $\mathrm{HFB}$ result $r_{\mathrm{rms}}^{\mathrm{HFB}}=5.05 \mathrm{fm}$ at $\eta=0.6$. From the exact nopairing (HF) case to the small pairing (HFB) case $(\eta<1.0)$, the decrease of the neutron rms radius is mainly due to the shrinkage of the rms radius of the occupied single-particle orbits $\left(3 p_{3 / 2}\right)$, which seems to coincide with the so-called pairing antihalo effect.

However, if we examine another example, ${ }^{122} \mathrm{Zr}$, we cannot find such a pairing antihalo effect at all. Here, we do not claim ${ }^{122} \mathrm{Zr}$ is a real halo nucleus. We will check the change of the rms radius with the increasing pairing strength. The total energy and the neutron rms radius are shown in Fig. 5(a) as a function of the pairing strength factor $\eta$. It is clearly seen that as the nucleus becomes more bound with stronger pairing correlations, the neutron rms radius first remains unchanged and then monotonically increases. In panel (b) we show the corresponding occupation probabilities of the $3 p, 2 f$, and $1 h$ orbits, coming from the quasiparticle states within $0 \leqslant E \leqslant$ $7 \mathrm{MeV}$ as a function of the pairing strength factor $\eta$. It is clear that, without pairing, the $1 h_{11 / 2}$ orbit is fully occupied. For a small pairing strength, the energy gap around $3 \mathrm{MeV}$ [see Fig. 3(a)] makes it difficult to scatter neutrons to higher orbits. Therefore the HFB result for the rms radius remains almost the same for all $\eta$ values $0 \leqslant \eta \leqslant 1.0$, similar to the HF case with a vanishing pairing energy. For a further increase of the pairing strength, the rms radius of the occupied $1 h_{11 / 2}$ orbit remains almost the same [see Fig. 3(c)]. However, the neutrons begin to occupy the $3 p$ and $2 f$ orbits, which can contribute a larger rms radius $r_{n l j}^{\mathrm{rms}}$ as shown in Fig. 3(c). Therefore in this case, we can

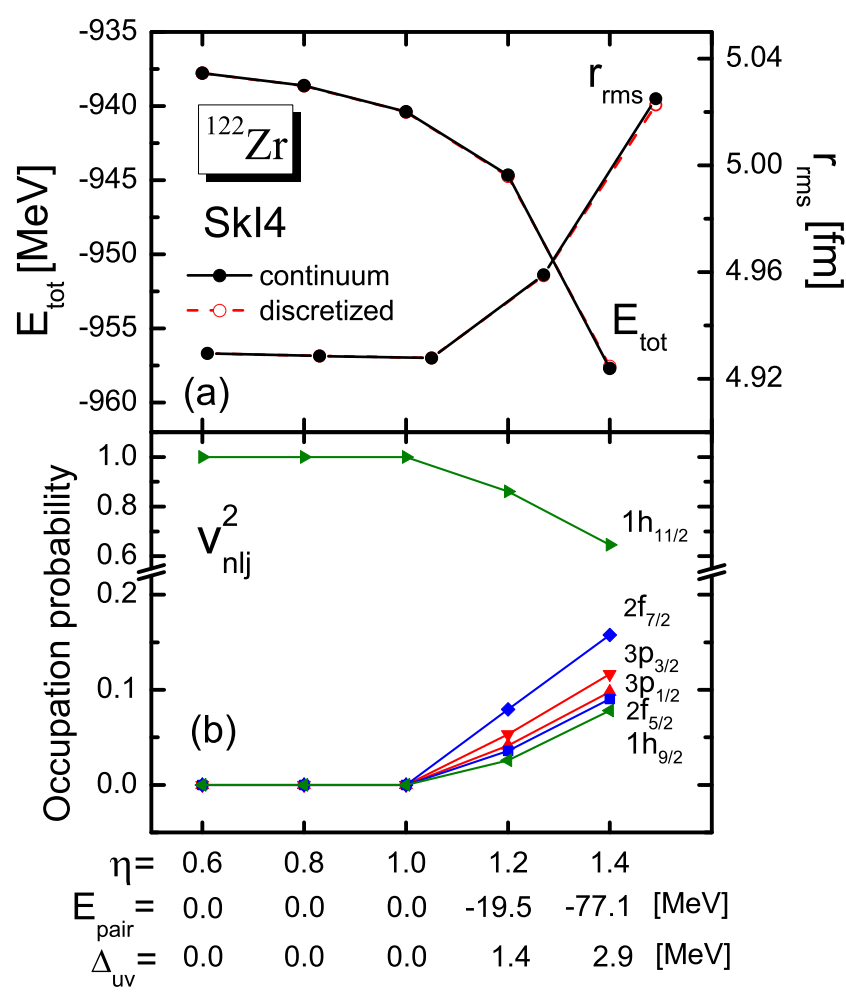

FIG. 5. (a) Total energy $E_{\text {tot }}$ and the neutron rms radius $r_{\text {rms }}$ obtained by the continuum (filled circle) and the discretized (open circle) HFB calculations for ${ }^{122} \mathrm{Zr}$, and (b) occupation probabilities of the $3 p, 2 f$, and $1 h$ orbits around the Fermi energy contributed from the quasiparticle states within $E=0-7 \mathrm{MeV}$ obtained by the continuum HFB calculation with the SkI4 interaction and the DDDI pairing force with different strengths $\eta V_{0}$.

only observe an expansion but no shrinkage of the neutron rms radius from the no-pairing to the finite pairing case. Actually, the difference between ${ }^{124} \mathrm{Zr}$ and ${ }^{122} \mathrm{Zr}$ is whether the weakly bound $3 p_{3 / 2}$ orbit is originally occupied or not. The asymptotic behavior of this wave function is sensitive to the pairing gap.

Finally, we would like to emphasize that the terminology "pairing antihalo effect" has to be taken with great care. In the original literatures [10], the pairing antihalo effect actually refers to the fact that the divergence of the HF wave function without pairing at the limit $\varepsilon \rightarrow 0$ can be avoided in the HFB wave function with finite pairing gap. It is restricted to the rather academic case of one fully occupied single-particle wave functions with low- $\ell$ value very close to the continuum limit. The realistic case is much more complicated. In particular one has to consider contributions of several partially occupied orbits and the occupation are determined by the pairing correlations. This can lead to a decrease or an increase of the various contributions. In some cases it is just the influence of pairing, which causes an extension of the neutron radius in such nuclei.

\section{CONCLUSIONS}

In the present investigations, we concentrated on the influence of pairing correlations on the size of the neutron-rich 
nuclei using a fully self-consistent description as well for the mean potential as for the pairing correlations. Taking the nucleus ${ }^{124} \mathrm{Zr}$ as an example, we performed a numerical analysis by self-consistent Skyrme HFB calculations varying the strength of pairing in reasonable limits. The continuum is treated appropriately by Green's function techniques. We find that the neutron rms radius first shrinks and then expands as the pairing strength increases. The expansion of the neutron rms radius by pairing seems to contradict to the so-called pairing antihalo effect, which is associated with a reduction of the halo size by the pairing correlations.

In fact, it is clear that stronger pairing causes the density of specific orbits to decay faster in the asymptotic region, especially for occupied low angular momentum states. This can lead to a shrinkage of the total rms radius. However, at the same time, one should also take into account the reoccupation processes caused by pairing and the corresponding coupling to the continuum. There exist "halo" orbits, i.e., weakly bound states and resonant states embedded in the continuum which, by themselves, have a large rms radius. Without pairing they are unoccupied and cannot contribute to the total radius. On the other hand the self-consistent solution of the corresponding HFB equations in the continuum leads to changes in the occupation pattern. This will lead to an increasing of the radius, which would not exist without pairing.
In the self-consistent calculation of our example nucleus ${ }^{124} \mathrm{Zr}$, the above two aspects compete with each other and cause with increasing pairing strength the total neutron rms radius first to decrease and then to increase. The terminology "pairing antihalo effect" only emphasizes the first aspect. Therefore, this terminology is somehow misleading for this purpose. After all, "halo" is not equivalent to "divergence," and pairing correlations play an important role in the anomalous increase of neutron radii in such nuclei.

\section{ACKNOWLEDGMENTS}

The author Y.Z. would like to thank L. L. Li and S. G. Zhou for helpful discussions. This work was partly supported by the Major State 973 Program2007CB815000; the National Natural Science Foundation of China under Grants No. 11405116, No. 11335002, and No. 11175002; the Research Fund for the Doctoral Program of Higher Education Grant No. 20110001110087; and the Oversea Distinguished Professor Project from Ministry of Education Grant No. MS2010BJDX001. We also acknowledge partial support from the DFG Cluster of Excellence "Origin and Structure of the Universe" (http://www.universe-cluster.de).
[1] D. M. Brink and R. A. Broglia, The Nuclear Superfluidity Pairing in Finite Systems (University Press, Cambridge, UK, 2005).

[2] G. F. Bertsch and H. Esbensen, Ann. Phys. (N.Y.) 209, 327 (1991).

[3] P. G. Hansen and B. Jonson, Europhys. Lett. 4, 409 (1987).

[4] J. Meng and P. Ring, Phys. Rev. Lett. 77, 3963 (1996).

[5] J. Meng and P. Ring, Phys. Rev. Lett. 80, 460 (1998).

[6] J. Meng, Nucl. Phys. A 635, 3 (1998).

[7] I. Tanihata, H. Hamagaki, O. Hashimoto, Y. Shida, N. Yoshikawa, K. Sugimoto, O. Yamakawa, T. Kobayashi, and N. Takahashi, Phys. Rev. Lett. 55, 2676 (1985).

[8] G. A. Lalazissis, D. Vretenar, W. Pöschl, and P. Ring, Nucl. Phys. A 632, 363 (1998).

[9] Y. Chen, P. Ring, and J. Meng, Phys. Rev. C 89, 014312 (2014).

[10] K. Bennaceur, J. Dobaczewski, and M. Ploszajczak, Phys. Lett. B 496, 154 (2000).

[11] P. Ring and P. Schuck, The Nuclear Many-Body Problem (Springer-Verlag, Berlin, 1980).

[12] M. Yamagami, Phys. Rev. C 72, 064308 (2005).

[13] S. Mizutori, J. Dobaczewski, G. A. Lalazissis, W. Nazarewicz, and P.-G. Reinhard, Phys. Rev. C 61, 044326 (2000).

[14] V. Rotival and T. Duguet, Phys. Rev. C 79, 054308 (2009).
[15] V. Rotival, K. Bennaceur, and T. Duguet, Phys. Rev. C 79, 054309 (2009).

[16] M. Bender, P.-H. Heenen, and P.-G. Reinhard, Rev. Mod. Phys. 75, 121 (2003).

[17] S. T. Belyaev, A. V. Smirnov, S. V. Tolokonnikov, and F. S. A., Sov. J. Nucl. Phys. 45, 783 (1987).

[18] M. Matsuo, Nucl. Phys. A 696, 371 (2001).

[19] Y. Zhang, M. Matsuo, and J. Meng, Phys. Rev. C 83, 054301 (2011).

[20] S. Q. Zhang, J. Meng, and S.-G. Zhou, Sci. China Phys. Mech. Astron. 46, 632 (2003).

[21] M. Grasso, S. Yoshida, N. Sandulescu, and N. Van Giai, Phys. Rev. C 74, 064317 (2006).

[22] Y. Zhang, M. Matsuo, and J. Meng, Phys. Rev. C 86, 054318 (2012).

[23] P.-G. Reinhard and H. Flocard, Nucl. Phys. A 584, 467 (1995).

[24] J. C. Pei, F. R. Xu, and P. D. Stevenson, Nucl. Phys. A 765, 29 (2006).

[25] M. Matsuo, Phys. Rev. C 73, 044309 (2006).

[26] M. Matsuo and Y. Serizawa, Phys. Rev. C 82, 024318 (2010).

[27] J. Dobaczewski, W. Nazarewicz, T. R. Werner, J. F. Berger, C. R. Chinn, and J. Dechargé, Phys. Rev. C 53, 2809 (1996). 